\title{
Generating Rotating Magnetic Fields With a Single Permanent Magnet for Propulsion of Untethered Magnetic Devices in a Lumen
}

\author{
Arthur W. Mahoney, Student Member, IEEE, and Jake J. Abbott, Member, IEEE
}

\begin{abstract}
To date, untethered magnetic devices actuated with a single rotating permanent magnet, such as active capsule endoscopes and magnetic microrobots, have been constrained to operate in positions where the rotating dipole field behavior is simple and easy to visualize. In this paper, we show how to generate a rotating magnetic field with any desired rotation axis, for magnetic device actuation, at any device position in space using a single rotatingmagnet actuator. The methods presented can control untethered rotating magnetic devices, while the rotating actuator magnet follows trajectories independent of the untethered devices themselves. We demonstrate our methods by actuating rotating magnetic devices in a lumen. Applications include minimally invasive medical tasks requiring an untethered magnetic device to operate in natural lumen pathways of the body (e.g., the gastrointestinal system, the subarachnoid space of the nervous system, or vasculature).
\end{abstract}

Index Terms-Capsule endoscopy, magnetic manipulation, medical robotics, microrobotics.

\section{INTRODUCTION}

$\mathbf{U}$ NTETHERED magnetic devices (UMDs), such as magnetic microrobots [1] and magnetically actuated capsule endoscopes [2], have become an active area of research because of their potential impact on minimally invasive medicine. These devices derive their power from externally applied magnetic fields. Some forms of actuation utilize magnetic forces for dragging [3]-[7], others apply magnetic torque to roll on a surface [8]-[10], swim through a fluid or lumen via helical screw propulsion [11]-[15], screw through soft tissue [16], [17], or swim with a flexible tail [18]. Because these devices range in size from the microscale to the mesoscale and employ many forms of magnetic propulsion, we refer to them herein as UMDs without any implied size, propulsion method, or application. These devices can be viewed as simple end-effectors of a larger robotic system.

Due to the ability of permanent magnets to generate strong magnetic fields at low cost, researchers are considering

Manuscript received June 6, 2013; accepted October 22, 2013. Date of publication November 21, 2013; date of current version April 1, 2014. This paper was recommended for publication by Associate Editor N. Simaan and Editor B. J. Nelson upon evaluation of the reviewers' comments. This work was supported by the National Science Foundation under Grant 0952718 and Grant 0654414.

A. W. Mahoney is with the School of Computing, University of Utah, Salt Lake City, UT 84112 USA (e-mail: art.mahoney@utah.edu).

J. J. Abbott is with the Department of Mechanical Engineering, University of Utah, Salt Lake City, UT 84112 USA (e-mail: jake.abbott@utah.edu).

This paper has supplementary multimedia (movie) downloadable material available at http://ieeexplore.ieee.org. The file size is $5.1 \mathrm{Mb}$.

Color versions of one or more of the figures in this paper are available online at http://ieeexplore.ieee.org.

Digital Object Identifier 10.1109/TRO.2013.2289019

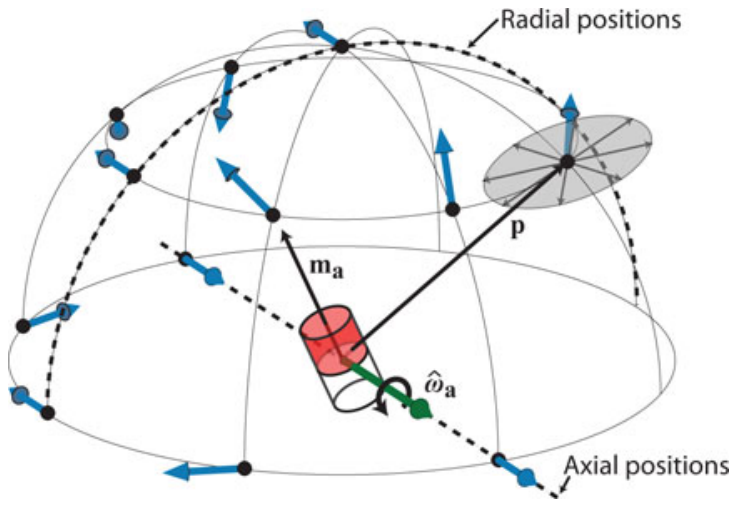

Fig. 1. When an actuator magnet with moment $\mathbf{m}_{\mathbf{a}}$ rotates around the axis $\hat{\boldsymbol{\omega}}_{\mathrm{a}}$ with $\mathbf{m}_{\mathrm{a}}$ perpendicular to $\hat{\boldsymbol{\omega}}_{\mathrm{a}}$, the dipole field vector $\mathbf{h}$ at any given position rotates around, and is perpendicular to, a constant axis $\hat{\omega}_{\mathrm{h}}$. The axis $\hat{\boldsymbol{\omega}}_{\mathrm{h}}$ at various positions are illustrated with large blue arrows. A representation of the ellipse traced out by the rotating magnetic field at the position $\mathbf{p}$ is shown. Any position on the $\hat{\omega}_{\mathrm{a}}$ axis is denoted to be in an axial position and any position in the plane spanned by the rotating $\mathbf{m}_{\mathbf{a}}$ is a radial position. The magnetic field at $\mathrm{p}$ is illustrated by gray arrows rotating around $\hat{\omega}_{\mathrm{h}}$.

permanent magnet systems for UMD actuation [8]-[12]. Actuation of UMDs using permanent magnets tends to be nontrivial due to the complex nature of the generated magnetic fields. In the case of actuation using a single permanent magnet, UMD control strategies developed to date have employed either attractive magnetic force or magnetic torque in simple ways for propulsion. Strategies exclusively employing attractive magnetic force, generated between a single permanent magnet and a UMD, employ the magnetic force for dragging [3], [4], [6], [7]. Strategies exclusively employing magnetic torque typically apply rotating magnetic fields, generated by a single rotating permanent magnet, that cause the UMD to rotate; UMD rotation is then transformed into propulsion using a helix or screw [11], [12], or by rolling [8]-[10].

Strategies that employ the magnetic force generated by a single permanent magnet tend to be limited by the fact that the force magnitude decreases faster than that of magnetic torque with increasing UMD-actuator separation distance, requiring either a large actuating magnet or performing actuation with the permanent magnet placed close to the UMD. Strategies that employ magnetic torque generated by a single rotating permanent magnet tend to be limited by the complexity of the rotating dipole field generated by the magnet. To simplify control, rotating UMDs have been exclusively operated in radial or axial positions relative to the rotating magnet (see Fig. 1) [8]-[12]. In these two positions, the rotating magnetic field $\mathbf{h}$ applied to 
the UMD rotates around an axis $\hat{\boldsymbol{\omega}}_{\mathrm{h}}$ that lies parallel to the actuator magnet's rotation axis $\hat{\omega}_{\mathrm{a}}$, making it easy to visualize the coupling between the rotating actuator magnet and the UMD. Requiring UMDs to be exclusively operated in these two positions, however, significantly constrains the physical placement of the actuator magnet.

It has been shown that when a dipole (e.g., rotating permanent magnet) rotates such that its magnetic moment is perpendicular to its rotation axis, the generated field at any position in space rotates around, and is perpendicular to, a constant axis, and its field magnitude is described by an ellipse (see Fig. 1) [19]. In this paper, we reformulate this phenomenon in a manner that readily enables a unique rotation axis of the actuator magnet to be found that produces any desired magnetic field rotation axis (which may be specifically required to produce rotating UMD propulsion) at any UMD position. This removes the need for rotating UMDs to be actuated exclusively in axial and radial positions, enabling the actuator magnet's position to move freely during UMD operation to avoid obstacles in the workspace, improve control authority, and potentially employ attractive magnetic force to simultaneously contribute to propulsion.

We demonstrate our results by measuring the rotating magnetic field, and driving a threaded-capsule UMD and rolling a spherical UMD through a lumen, using a rotating permanent magnet (which we will refer to as the "actuator magnet") positioned in space by a 6-degrees-of-freedom (DOF) robotic manipulator. Applications include any minimally invasive medical task that requires a UMD to operate in the natural lumen pathways of the body (e.g., the gastrointestinal (GI) tract, the subarachnoid space of the nervous system, or vasculature). Robotic systems that apply our method for actuating UMDs will be more flexible in their choice of actuator-magnet position.

This paper contains results that first appeared in [20], as well as additional formal analysis of magnetic field properties, experimental results, and discussion. The experimental results presented are proof-of-concept experiments that confirm the theory contained herein. The problem of finding the optimal actuatormagnet position for UMD actuation, the best choice of localization system, and the magnetic fields necessary for propulsion in the human GI tract is beyond this paper's scope.

\section{Controlling Rotating Untethered Magnetic Devices With a Single Rotating Permanent Magnet}

In this paper, scalars are denoted by lower-case standard font (e.g., c), vectors are denoted by lower-case bold font (e.g., x), and matrices are denoted by capital standard font (e.g., $M$ ). The symbol denotes a unit-length vector (e.g., $\hat{\mathbf{x}}$ ).

Let the dipole moment of the UMD's magnet be $\mathbf{m}_{\mathbf{d}} \in$ $\mathbb{R}^{3}\left\{\mathrm{~A} \cdot \mathrm{m}^{2}\right\}$. If a magnetic field $\mathbf{h} \in \mathbb{R}^{3}\left\{\mathrm{~A} \cdot \mathrm{m}^{-1}\right\}$ is applied to the UMD, then a magnetic torque $\tau\{\mathrm{N} \cdot \mathrm{m}\}$ and a magnetic force $f\{N\}$ will be produced:

$$
\begin{aligned}
\boldsymbol{\tau} & =\mu_{0} \mathbf{m}_{\mathbf{d}} \times \mathbf{h} \\
\mathbf{f} & =\mu_{0}\left(\mathbf{m}_{\mathbf{d}} \cdot \nabla\right) \mathbf{h}
\end{aligned}
$$

where $\mu_{0}=4 \pi \times 10^{-7} \mathrm{~N} \cdot \mathrm{A}^{-2}$ is the permeability of free space [21]. Note that in the literature, the magnetic field is typically denoted as $\mathbf{H}$, however, we denote it in lower-case as $\mathbf{h}$ to maintain the conventions of this paper.

The magnetic torque causes $\mathbf{m}_{\mathbf{d}}$ to rotate in the direction of $\mathbf{h}$. If the magnetic field $\mathbf{h}$ rotates around an axis $\hat{\boldsymbol{\omega}}_{\mathbf{h}}$ (with direction of rotation given by the "right-hand" rule), then $\tau$ will cause $\mathbf{m}_{\mathbf{d}}$ (and thus the UMD) to continuously rotate. The magnetic force $\mathbf{f}$ causes the UMD to translate in a direction determined by the UMD's dipole moment $\mathbf{m}_{\mathbf{d}}$ and the spatial derivative of the magnetic field. UMD propulsion can be produced using the magnetic torque to generate UMD rotation, which is converted into propulsion via rolling or with a screw thread, the magnetic force can be employed for pulling, or both can be used in concert.

If the magnetic field $\mathbf{h}$ is generated using a single actuator magnet, then its field at the UMD's position p (see Fig. 1), relative to the actuator-magnet center, can be approximated by the point-dipole model

$$
\mathbf{h}=\frac{1}{4 \pi\|\mathbf{p}\|^{3}} H \mathbf{m}_{\mathbf{a}}
$$

where $\mathbf{m}_{\mathbf{a}} \in \mathbb{R}^{3}\left\{\mathrm{~A} \cdot \mathrm{m}^{2}\right\}$ is the dipole moment of the actuator magnet, $H=3 \hat{\mathbf{p}} \hat{\mathbf{p}}^{\top}-I$, and $I \in \mathbb{R}^{3 \times 3}$ is the identity matrix [21]. Equation (3) exactly predicts the field produced by a spherical magnet. For all other geometries, it is an approximation that becomes more accurate with increasing distance. A nonspherical geometry can be chosen to be well approximated by (3) at smaller distances [22].

\section{A. Rotation Axis of the Magnetic Field}

For UMDs that employ magnetic torque generated by the rotating magnetic field $\mathbf{h}$ for propulsion, appropriately selecting the rotation axis $\hat{\omega}_{\mathrm{h}}$ of the magnetic field is a critical component of the control strategy. For UMDs in free medium (e.g., helical microswimmers in fluid) or those that roll, the UMD rotation axis naturally aligns itself with $\hat{\boldsymbol{\omega}}_{\mathrm{h}}$ and varying $\hat{\boldsymbol{\omega}}_{\mathrm{h}}$ steers the UMD. For screw-like UMDs constrained in a lumen (e.g., a magnetic capsule endoscope in the small intestine), $\hat{\omega}_{\mathrm{h}}$ should be locally aligned with the lumen in order to apply the most useful magnetic torque.

1) Choosing $\hat{\boldsymbol{\omega}}_{\mathrm{a}}$ Given $\hat{\boldsymbol{\omega}}_{\mathrm{h}}$ and $\hat{\mathbf{p}}$ : For a dipole field generated by an actuator magnet that rotates around the axis $\hat{\boldsymbol{\omega}}_{\mathrm{a}}$, the required rotation axis $\hat{\omega}_{\mathrm{a}}$ to make the magnetic field $\mathbf{h}$ at any desired position $\mathbf{p}$ rotate around a desired axis $\hat{\omega}_{\mathrm{h}}$ is not easy to visualize. If the actuator magnet is rotated such that its dipole moment $\mathbf{m}_{\mathbf{a}}$ is perpendicular to $\hat{\boldsymbol{\omega}}_{\mathrm{a}}$ (i.e., $\mathbf{m}_{\mathrm{a}}^{\top} \hat{\boldsymbol{\omega}}_{\mathrm{a}}=0$ ) and it is desired that the field $\mathbf{h}$ be perpendicular to $\hat{\omega}_{\mathrm{h}}$ (i.e., $\left.\mathbf{h}^{\top} \hat{\boldsymbol{\omega}}_{\mathbf{h}}=0\right)$, then the necessary $\hat{\boldsymbol{\omega}}_{\mathrm{a}}$ given a desired $\hat{\boldsymbol{\omega}}_{\mathbf{h}}$ can be found by substituting (3) into the projection $\mathbf{h}^{\top} \hat{\boldsymbol{\omega}}_{\mathbf{h}}$ and applying the symmetry of $H$ to produce the expression

$$
\mathbf{h}^{\top} \hat{\omega}_{\mathbf{h}}=\frac{1}{4 \pi\|\mathbf{p}\|^{3}} \mathbf{m}_{\mathrm{a}}^{\top} H \hat{\boldsymbol{\omega}}_{\mathbf{h}}=0 .
$$

Because $H \hat{\omega}_{\mathrm{h}}$ does not vary as the actuator magnet rotates, and because $\mathbf{m}_{\mathrm{a}}^{\top} \hat{\boldsymbol{\omega}}_{\mathrm{a}}=0$, letting $\hat{\boldsymbol{\omega}}_{\mathrm{a}}$ lie parallel to $H \hat{\boldsymbol{\omega}}_{\mathrm{h}}$ is the only solution for $\hat{\omega}_{\mathrm{a}}$ that satisfies (4) and is simultaneously invariant to the rotation of $\mathbf{m}_{\mathbf{a}}$. Therefore, given $\hat{\boldsymbol{\omega}}_{\mathrm{h}}$ and the UMD position $\mathrm{p}$, the necessary actuator-magnet rotation axis $\hat{\omega}_{\mathrm{a}}$ can be found 
with

$$
\hat{\omega}_{\mathrm{a}}=\widehat{H \hat{\omega}_{\mathrm{h}}} \text {. }
$$

Because $H$ is constructed using $\hat{\mathbf{p}}, H$ only varies with changes in the direction of $\hat{\mathbf{p}}$. This implies that solutions obtained from (5) are invariant to scaling $\|\mathbf{p}\|$ by moving the actuator magnet nearer to or farther away from the UMD, provided that the direction of $\mathbf{p}$ remains unchanged. Naturally, the magnitude of the field will depend on $\|\mathbf{p}\|$. In addition, it is shown in Appendix A that $\mathbf{p}, \hat{\boldsymbol{\omega}}_{\mathrm{h}}$, and $\hat{\boldsymbol{\omega}}_{\mathrm{a}}$ are always coplanar.

It can be easily verified using (3) that the eigenvectors of $H$ include $\hat{\mathbf{p}}$ and a two-dimensional eigenspace consisting of vectors in the plane orthogonal to $\hat{\mathbf{p}}$, with corresponding eigenvalues $\lambda_{1}=2$ and $\lambda_{2}=\lambda_{3}=-1$, respectively. Because $\operatorname{det}(H)=\lambda_{1} \lambda_{2} \lambda_{3}=2$ for all $\mathbf{p}, H$ is always invertible and there exists exactly one actuator-magnet rotation axis $\hat{\omega}_{\mathrm{a}}$ to generate a desired field rotation about the axis $\hat{\omega}_{\mathrm{h}}$ for any UMD position $\mathbf{p}$. The forward problem, which gives the local field axis of rotation $\hat{\omega}_{\mathrm{h}}$ at the position $\mathbf{p}$, given the actuator magnet's axis of rotation $\hat{\omega}_{\mathrm{a}}$, is found with

$$
\hat{\omega}_{\mathrm{h}}=\widehat{H^{-1} \hat{\omega}_{\mathrm{a}}}
$$

where $H^{-1}=(H-I) / 2$ (derived in Appendix B). Fig. 1 shows $\hat{\omega}_{\mathrm{h}}$ at positions on a half-hemisphere with a given $\hat{\omega}_{\mathrm{a}}$.

With $\mathbf{p}$ obtained from a localization system and the desired applied field rotation axis $\hat{\omega}_{\mathrm{h}}$ known, the necessary actuatormagnet axis of rotation $\hat{\omega}_{\mathrm{a}}$ can be quickly found by (5). As the UMD or the actuator magnet moves and the direction of p changes, (5) must be updated by repacking the matrix $H$, and a new solution $\hat{\omega}_{\mathrm{a}}$ must be produced to maintain the desired applied field rotation axis at p. Theoretically, a desired actuator-magnet position trajectory can be first established and the necessary $\hat{\omega}_{\mathrm{a}}$ can be found during execution given the position of the actuator magnet and the UMD. In practice, however, the actuator magnet's position trajectory should adapt to the UMD's behavior in order to maintain control authority while simultaneously avoiding hardware collisions or other constraints. If the actuator magnet is positioned using a robotic manipulator, every potential orientation of $\hat{\omega}_{\mathrm{a}}$ may not be physically achievable at every manipulator pose due to range constraints of the manipulator's joints. This issue may be alleviated during motion planning by utilizing the fact that a desired $\hat{\omega}_{\mathrm{a}}$ is achievable from at least two unique manipulator poses by reversing the actuator magnet's rotation direction.

2) Choosing $\hat{\mathbf{p}}$ Given $\hat{\omega}_{\mathrm{h}}$ and $\hat{\boldsymbol{\omega}}_{\mathrm{a}}$ : If the direction of the actuator magnet's rotation axis $\hat{\omega}_{\mathrm{a}}$ is fixed (e.g., if the actuator magnet is positioned using only a 3-DOF gantry robot), then any desired applied field rotation axis $\hat{\boldsymbol{\omega}}_{\mathrm{h}}$ can still be achieved at any UMD position by sacrificing actuator-magnet positioning flexibility and placing the actuator magnet in a specific position $\hat{\mathbf{p}}$. If the direction of $\hat{\boldsymbol{\omega}}_{\mathrm{a}}$ can be reversed by changing the actuator magnet's spin direction, then there exist at least four unique directions of position $\hat{\mathbf{p}}$ where the actuator magnet can be placed to achieve any desired $\hat{\omega}_{\mathrm{h}}$.

With the desired field rotation axis $\hat{\boldsymbol{\omega}}_{\mathrm{h}}$ given and the actuatormagnet rotation axis $\hat{\omega}_{\mathrm{a}}$ fixed and known, the solutions for the necessary actuator-magnet position (actually computed as the position direction $\hat{\mathbf{p}}$ of the UMD relative to the actuator magnet) are found using the projection $\gamma=\hat{\mathbf{p}}^{\top} \hat{\boldsymbol{\omega}}_{\mathrm{h}}$, obtained by first computing the intermediate projection

$$
\rho=\hat{\boldsymbol{\omega}}_{\mathrm{h}}^{\top} \hat{\boldsymbol{\omega}}_{\mathrm{a}}=\frac{\hat{\boldsymbol{\omega}}_{\mathbf{h}}^{\top} H \hat{\boldsymbol{\omega}}_{\mathrm{h}}}{\left\|H \hat{\boldsymbol{\omega}}_{\mathrm{h}}\right\|}=\frac{3 \gamma^{2}-1}{\sqrt{3 \gamma^{2}+1}}
$$

after substituting (5) for $\hat{\omega}_{\mathrm{a}}$. (The value of $\rho$ is known since $\hat{\omega}_{\mathrm{h}}$ and $\hat{\omega}_{\mathrm{a}}$ are given.) Squaring both sides of (7) and grouping terms produces a polynomial in terms of the unknown $\gamma$

$$
9 \gamma^{4}-3\left(2+\rho^{2}\right) \gamma^{2}+\left(1-\rho^{2}\right)=0 .
$$

There are two solutions of (8) for $\gamma^{2}$, which can be found using the quadratic formula

$$
\begin{aligned}
& \gamma_{+}^{2}=\frac{2+\rho^{2}+\sqrt{8 \rho^{2}+\rho^{4}}}{6} \geq \frac{1}{3} \\
& \gamma_{-}^{2}=\frac{2+\rho^{2}-\sqrt{8 \rho^{2}+\rho^{4}}}{6} \leq \frac{1}{3}
\end{aligned}
$$

and are verifiably bound by $1 / 3$ from below and above, respectively. After examining the right-hand side of (7), it is clear that $\rho \leq 0$ requires $\gamma^{2} \leq 1 / 3$ and $\rho \geq 0$ requires $\gamma^{2} \geq 1 / 3$. Given the lower and upper bounds of (9) and (10), respectively, the solution of (7) is

$$
\gamma^{2}=\left\{\begin{array}{ll}
\gamma_{+}^{2} & \text { if } \rho \geq 0 \\
\gamma_{-}^{2} & \text { if } \rho \leq 0
\end{array} .\right.
$$

For a value of $\gamma^{2}$ given by (11), two solutions for $\hat{\mathbf{p}}$ can be found (due to $\pm \gamma$ ) in opposite directions of each other: the solution where $\gamma \geq 0$ is denoted by $+\hat{\mathbf{p}}$ and the opposite solution where $\gamma \leq 0$ is denoted by $-\hat{\mathbf{p}}$. Using the fact that $\hat{\boldsymbol{\omega}}_{\mathrm{a}}, \hat{\boldsymbol{\omega}}_{\mathrm{h}}$, and $\pm \hat{\mathbf{p}}$ are always coplanar (see Appendix A), $+\hat{\mathbf{p}}$ can be constructed by first generating an orthonormal basis for the plane spanned by $\hat{\omega}_{\mathrm{a}}$ and $\hat{\omega}_{\mathrm{h}}$. After selecting the first basis vector to be $\hat{\omega}_{\mathrm{h}}$, a second basis vector $\hat{\omega}_{\mathrm{h}}^{\perp}$ is

$$
\hat{\omega}_{\mathrm{h}}^{\perp}=\frac{\left(I-\hat{\omega}_{\mathrm{h}} \hat{\omega}_{\mathrm{h}}^{\top}\right) \hat{\omega}_{\mathrm{a}}}{\left\|\left(I-\hat{\omega}_{\mathrm{h}} \hat{\omega}_{\mathrm{h}}^{\top}\right) \hat{\omega}_{\mathrm{a}}\right\|} .
$$

Since $\gamma$ is the projection of $\hat{\mathbf{p}}$ onto $\hat{\boldsymbol{\omega}}_{\mathrm{h}}$, the vector $+\hat{\mathbf{p}}$ can be formed from the orthonormal basis as

$$
+\hat{\mathbf{p}}=|\gamma| \hat{\boldsymbol{\omega}}_{\mathrm{h}}+\sqrt{1-|\gamma|^{2}} \hat{\boldsymbol{\omega}}_{\mathrm{h}}^{\perp}
$$

where $|\gamma|$ is obtained from (11), and the solution for $-\hat{\mathbf{p}}$ is given by $-1(+\hat{\mathbf{p}})$. After $\pm \hat{\mathbf{p}}$ is determined, $\|\mathbf{p}\|$ can be selected without changing the result of (13).

There are two cases when (12) becomes degenerate and the aforementioned approach for constructing $\pm \hat{\mathbf{p}}$ breaks down, both are illustrated in Fig. 2(a). The first case occurs when $\hat{\omega}_{\mathrm{h}}=\hat{\boldsymbol{\omega}}_{\mathrm{a}}$. In this case, it can be verified using (11) that $\gamma^{2}=1$ implying that $\pm \hat{\mathbf{p}}$ are parallel to $\hat{\boldsymbol{\omega}}_{\mathrm{h}}$ and $\hat{\boldsymbol{\omega}}_{\mathrm{a}}$. These positions correspond to axial positions (see Fig. 1). The second degenerate case occurs when $\hat{\omega}_{\mathrm{h}}=-\hat{\boldsymbol{\omega}}_{\mathrm{a}}$. In this case, $\gamma^{2}=0$ implying that $\pm \hat{\mathbf{p}}$ must be perpendicular to $\hat{\boldsymbol{\omega}}_{\mathrm{h}}$ and $\hat{\boldsymbol{\omega}}_{\mathrm{a}}$. There are an infinite number of solutions in this case, and all correspond to radial positions (see Fig. 1). Reversing the actuator magnet's spin direction turns one degenerate case into the other.

In every other nondegenerate case, spinning the actuator magnet about $\hat{\omega}_{\mathrm{a}}$ in one direction admits two unique solutions for $\hat{\mathbf{p}}$, and spinning in the opposite direction changes the sign of 

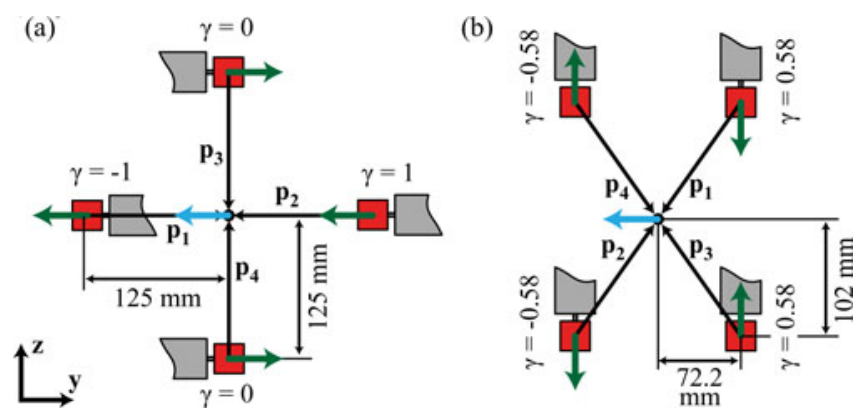

Fig. 2. (a) Configurations that cause (12) to become degenerate, for $\|\mathbf{p}\|=$ $125 \mathrm{~mm}$. (b) An example set of actuator-magnet position solutions that achieve a desired field rotation axis $\hat{\boldsymbol{\omega}}_{\mathrm{h}}=[0,-1,0]^{\mathrm{T}}$ when the actuator magnet's rotation axis is constrained to $\hat{\boldsymbol{\omega}}_{\mathrm{a}}=[0,0,-1]^{\top}$ and $\|\mathbf{p}\|=125 \mathrm{~mm}$. The positions $\mathbf{p}_{1}$ and $\mathbf{p}_{\mathbf{2}}$ are the $+\hat{\mathbf{p}}$ and $-\hat{\mathbf{p}}$ solutions, respectively, given by (13). If the actuator magnet's spin direction can be reversed such that $\hat{\boldsymbol{\omega}}_{\mathbf{a}}=[0,0,1]^{\top}$, then $\mathbf{p}_{3}$ and $\mathbf{p}_{4}$ are the corresponding $+\hat{\mathbf{p}}$ and $-\hat{\mathbf{p}}$ solutions.

$\rho$, produces another value of $\gamma^{2}$ from (11), and admits two additional unique solutions for $\hat{\mathbf{p}}$ making, in total, four unique solutions that produce a desired field rotation axis $\hat{\omega}_{\mathrm{h}}$ given a reversible actuator-magnet rotation axis $\hat{\omega}_{\mathrm{a}}$. Fig. 2(b) shows an example set of actuator-magnet positions that achieve a desired field rotation axis when the actuator magnet's rotation axis is constrained but is permitted to reverse direction.

\section{B. Magnitude and Rotation Speed of the Magnetic Field}

For rotating UMDs, a common failure mode that results in the loss of control authority occurs when the UMD steps out of synchronization with the rotating field as the field rotates. The rotation frequency above which the applied magnetic torque is too weak in magnitude to keep the UMD synchronized with the rotating field is referred to as the "step-out" frequency and is denoted by $\left\|\omega_{\text {so }}\right\|$. If a UMD's rotational dynamics are dominated by friction and inertia is negligible, then its angular velocity is approximately proportional to the magnetic torque by a linear damping coefficient $c$, and $\left\|\boldsymbol{\omega}_{\text {so }}\right\|$ is the speed that requires the total available magnetic torque to maintain synchronization (a similar property holds for UMDs with nonnegligible inertia). The maximum available torque at any instant in time is $\|\boldsymbol{\tau}\|_{\max }=\mu_{0}\left\|\mathbf{m}_{\mathbf{d}}\right\|\|\mathbf{h}\|$ resulting in the step-out frequency $\left\|\boldsymbol{\omega}_{\text {so }}\right\|=\|\boldsymbol{\tau}\|_{\max } / c$.

In rotating nonuniform fields produced by an actuator magnet, the applied field magnitude and angular velocity, at any given UMD position $\mathbf{p}$, depend on the orientation of $\mathbf{m}_{\mathbf{a}}$ and $\mathbf{p}$. During actuator-magnet rotation, the instantaneous field magnitude $\|\mathbf{h}\|$ fluctuates in an elliptical fashion, and is

$$
\|\mathbf{h}\|=\frac{\left\|\mathbf{m}_{\mathbf{a}}\right\|}{4 \pi\|\mathbf{p}\|^{3}} \sqrt{1+3\left(\hat{\mathbf{m}}_{\mathrm{a}}^{\top} \hat{\mathbf{p}}\right)^{2}}
$$

and the minimum and maximum field magnitudes are

$$
\begin{aligned}
\|\mathbf{h}\|_{\min } & =\frac{\left\|\mathbf{m}_{\mathbf{a}}\right\|}{4 \pi\|\mathbf{p}\|^{3}} \\
\|\mathbf{h}\|_{\max } & =\frac{\left\|\mathbf{m}_{\mathbf{a}}\right\|}{4 \pi\|\mathbf{p}\|^{3}} \sqrt{1+3\|\tilde{\mathbf{p}}\|^{2}}
\end{aligned}
$$

where the vector $\tilde{\mathbf{p}}=\left(I-\hat{\boldsymbol{\omega}}_{\mathrm{a}} \hat{\boldsymbol{\omega}}_{\mathrm{a}}^{\top}{ }^{\top}\right) \hat{\mathbf{p}}$ is the projection of $\hat{\mathbf{p}}$ onto the plane perpendicular to $\hat{\boldsymbol{\omega}}_{\mathrm{a}}$ (the plane in which $\mathbf{m}_{\mathbf{a}}$ resides). The maximum field magnitude occurs when $\mathbf{m}_{\mathbf{a}}$ is parallel to $\tilde{\mathbf{p}}$, and the minimum field magnitude occurs when $\mathbf{m}_{\mathbf{a}}$ is perpendicular to $\tilde{\mathbf{p}}$. In the special case of $\hat{\boldsymbol{\omega}}_{\mathrm{a}}=\hat{\mathbf{p}}$, corresponding to the axial positions that are depicted in Fig. 1, then $\tilde{\mathbf{p}}=0$ and $\|\mathbf{h}\|_{\min }=\|\mathbf{h}\|_{\max }$.

The instantaneous angular velocity of the applied field, as it rotates around $\hat{\omega}_{\mathrm{h}}$, varies through each actuator-magnet cycle as well. If $\boldsymbol{\omega}_{\mathrm{h}}$ and $\boldsymbol{\omega}_{\mathrm{a}}$ denote the instantaneous angular velocities of the applied field and the actuator magnet, respectively, then the relation between $\left\|\omega_{\mathrm{h}}\right\|$ and $\left\|\boldsymbol{\omega}_{\mathrm{a}}\right\|$ is given by

$$
\left\|\boldsymbol{\omega}_{\mathbf{h}}\right\|=\left(\frac{\|\mathbf{h}\|_{\min }\|\mathbf{h}\|_{\max }}{\|\mathbf{h}\|^{2}}\right)\left\|\boldsymbol{\omega}_{\mathbf{a}}\right\| .
$$

Because $\|\mathbf{h}\|>0$ at all times, it is clear that the maximum angular velocity of the applied field occurs at the instant when the magnetic field strength $\|\mathbf{h}\|$ is the weakest, and the minimum angular velocity occurs when $\|\mathbf{h}\|$ is the strongest.

Because rotating the field faster than $\left\|\boldsymbol{\omega}_{\text {so }}\right\|$ causes the UMD to become unsynchronized with the field, $\left\|\omega_{\mathrm{a}}\right\|$ should be limited so that $\left\|\omega_{\mathrm{h}}\right\| \leq\left\|\boldsymbol{\omega}_{\text {so }}\right\|$ for all time. Since both the magnitude and angular velocity of the rotating field vary through each cycle, the maximum actuator-magnet rotation speed that guarantees synchronization also varies through each cycle. The maximum actuator speed can be found using

$$
\left\|\boldsymbol{\omega}_{\mathbf{h}}\right\| \leq\left\|\boldsymbol{\omega}_{\mathrm{so}}\right\|=\frac{\|\boldsymbol{\tau}\|_{\max }}{c}=\frac{\mu_{0}\left\|\mathbf{m}_{\mathbf{d}}\right\|\|\mathbf{h}\|}{c} .
$$

Substituting (17) for $\left\|\boldsymbol{\omega}_{\mathrm{h}}\right\|$, then solving for $\left\|\boldsymbol{\omega}_{\mathrm{a}}\right\|$ produces

$$
\left\|\boldsymbol{\omega}_{\mathbf{a}}\right\| \leq \frac{\mu_{0}\left\|\mathbf{m}_{\mathbf{d}}\right\|\|\mathbf{h}\|^{3}}{c\|\mathbf{h}\|_{\min }\|\mathbf{h}\|_{\max }} .
$$

The fastest constant actuator-magnet angular velocity that satisfies (19) can be found as

$$
\left\|\boldsymbol{\omega}_{\mathbf{a}}\right\|=\frac{\mu_{0}\left\|\mathbf{m}_{\mathbf{d}}\right\|\|\mathbf{h}\|_{\min }^{2}}{c\|\mathbf{h}\|_{\max }}
$$

after substituting $\|\mathbf{h}\|_{\text {min }}$ for $\|\mathbf{h}\|$ in (19).

If desired, the step-out frequency can be increased by moving the rotating actuator magnet closer to the UMD (i.e., decreasing $\|\mathbf{p}\|$ and increasing $\|\mathbf{h}\|)$, without changing the solution for $\hat{\boldsymbol{\omega}}_{\mathbf{h}}$. This should be done with care, however, as decreasing $\|\mathbf{p}\|$ also increases the applied magnetic force. In most situations, it is likely that the friction coefficient $c$ will vary and will not be known a priori. For magnetic capsule endoscopy, Hall-effect sensors aboard a mockup capsule have been used to estimate the onset of step-out [23]. When step-out is detected, either the actuator magnet should adapt by moving closer to the capsule or by rotating slower.

\section{Applied Magnetic Force}

The actuator magnet's field causes a magnetic force to be applied to the UMD, which can be derived from (2) as

$$
\mathbf{f}=\frac{3 \mu_{0}}{4 \pi\|\mathbf{p}\|^{4}}\left(\hat{\mathbf{p}} \mathbf{m}_{\mathrm{d}}^{\top}+\mathbf{m}_{\mathbf{d}} \hat{\mathbf{p}}^{\top}+\left(\mathbf{m}_{\mathrm{d}}^{\top} \hat{\mathbf{p}}\right) Z\right) \mathbf{m}_{\mathbf{a}}=F \mathbf{m}_{\mathbf{a}}
$$


where the matrix $Z=I-5 \hat{\mathbf{p}} \hat{\mathbf{p}}^{\top}$. When actuated well beneath a UMD's step-out frequency, the magnetic force generally tends to attract the UMD toward the actuator magnet. For configurations where the actuator magnet is positioned in a way that attractive magnetic force has a component in the desired direction of propulsion, then the attractive force can be beneficial. If the actuator magnet is placed where the magnetic force has a component directed against the desired propulsion direction, then the attractive force hinders actuation.

UMDs that are able to convert a rotating magnetic field into propulsion can propel themselves against attractive forces so long as the propulsive force is larger than the magnetic force. In the case where the desired propulsion direction is against the attractive magnetic force, the rotation speed at which the UMD's propulsive force balances the attractive force is called the "break-away" speed [11]. If the UMD is actuated slower than its break-away speed, then the UMD will be attracted toward the actuator magnet. The attraction can become selfcompounding as the magnetic force increases dramatically (as $\|\mathbf{p}\|^{-4}$ according to (21)) with decreasing distance between the actuator magnet and the UMD. In this paper, the UMD is actuated at distances where the UMD's weight, friction, and the propulsive force overpower any attractive magnetic force.

The ability of a rotating UMD to propel against attractive magnetic forces increases flexibility in actuator-magnet placement compared with other propulsion strategies. Stably driving a UMD away from a single permanent magnet, when solely applying magnetic force for pushing actuation, is challenging because the magnetic torque tends to align the UMD's dipole moment $\mathbf{m}_{\mathbf{d}}$ such that the magnetic force is always attractive.

For clinical applications, it is likely that the magnetic force must be managed to ensure safety [4], [24]. It has been found that actuating a UMD at its step-out frequency, in any actuatormagnet position, reduces the magnitude of the force and redirects it such that no component of the average magnetic force applied to the UMD over one rotation cycle is attractive [24]. In the event of loss of UMD localization or loss of control authority, the actuator magnet's ability to apply undesirable magnetic force to a UMD can be dramatically reduced by rapidly increasing the separation distance $\|\mathbf{p}\|$, or by rotating the actuator magnet well above the UMD's step-out frequency. If the actuator magnet is rotated fast enough, the UMD's dipole moment $\mathbf{m}_{\mathbf{d}}$ will remain approximately stationary in space. Assuming $\mathbf{p}$ is approximately constant over one actuator-magnet revolution and the actuator magnet's rotation about $\hat{\omega}_{\mathrm{a}}$ is parameterized by the angle $\phi$, the averaged magnetic force applied to the UMD is then

$$
\overline{\mathbf{f}}=\frac{1}{2 \pi} \int_{0}^{2 \pi} F \mathbf{m}_{\mathbf{a}} d \phi=\frac{1}{2 \pi} F \int_{0}^{2 \pi} \mathbf{m}_{\mathbf{a}} d \phi=\mathbf{0}
$$

since $F$ is approximately constant and the rotation of $\mathbf{m}_{\mathrm{a}}$ about the axis $\hat{\boldsymbol{\omega}}_{\mathrm{a}}$ generates an odd function in $\mathbb{R}^{3}$, which integrates to zero over a complete actuator-magnet revolution.

Equation (22) will become more accurate as $\left\|\omega_{\mathbf{h}}\right\|$ increases above step-out. This is demonstrated in Fig. 3, which shows $\|\overline{\mathbf{f}}\|$ obtained from a simulation of a UMD whose dynamics are dominated by friction [and whose step-out frequency $\left\|\omega_{\text {so }}\right\|$ is

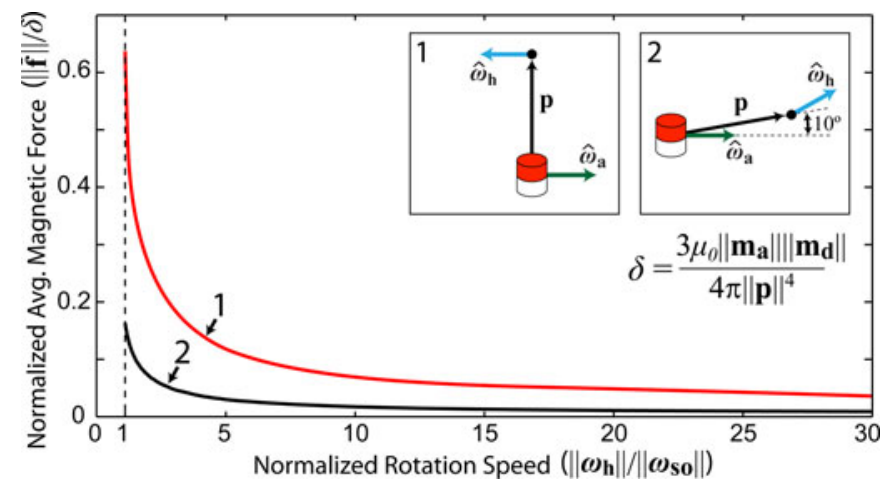

Fig. 3. The average magnetic force $\overline{\mathbf{f}}$ applied to a UMD over one actuatormagnet revolution asymptotically approaches $\mathbf{0}$ as the rotation frequency increases above step-out. Nondimensionalized simulation results of $\|\overline{\mathbf{f}}\|$ (normalized by $\delta$ ) are shown for a UMD whose dynamics are dominated by friction (the step-out frequency is given by (18)) as $\left\|\boldsymbol{\omega}_{\mathbf{h}}\right\|$ (normalized by $\left\|\boldsymbol{\omega}_{\text {so }}\right\|$ ) increases. Results in two UMD positions are shown.
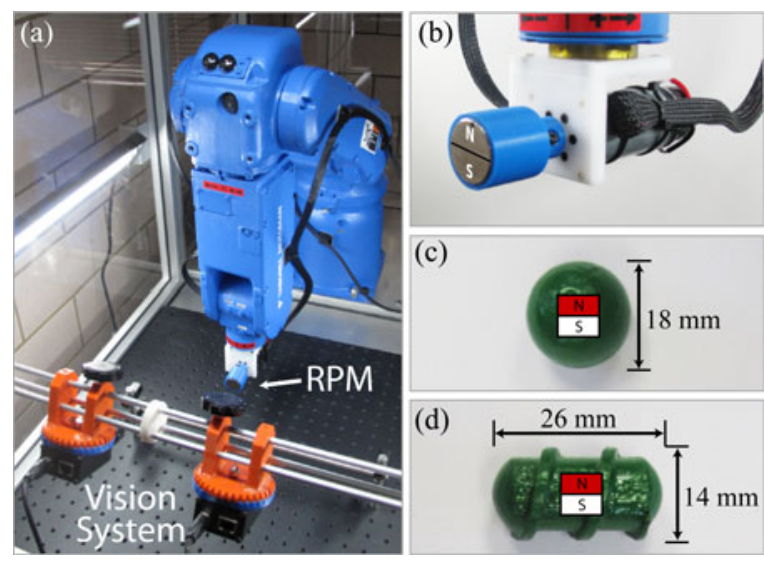

Fig. 4. The Yaskawa Motoman MH5 6-DOF robotic manipulator (a) is used to position the actuator magnet (b) for point-dipole calibration, and for actuating a spherical rolling UMD (c) and a threaded capsule-mockup UMD (d). The screw thread of the capsule UMD has a pitch of $7 \mathrm{~mm}$ and is $1.5 \mathrm{~mm}$ deep. Both devices contain a 6.35 - mm cube Grade-N52 NdFeB magnet.

given by (18)] in two positions relative to the actuator magnet. The results of Fig. 3 are nondimensionalized by scaling $\|\overline{\mathbf{f}}\|$ by $\delta=3 \mu_{0}\left\|\mathbf{m}_{\mathbf{d}}\right\|\left\|\mathbf{m}_{\mathbf{a}}\right\| /\left(4 \pi\|\mathbf{p}\|^{4}\right)$ to remove the influence of $\|\mathbf{p}\|,\left\|\mathbf{m}_{\mathbf{d}}\right\|$, and $\left\|\mathbf{m}_{\mathbf{a}}\right\|$, and by scaling $\left\|\omega_{\mathbf{h}}\right\|$ by the UMD's step-out frequency to remove the influence of friction $c$. The results in two UMD positions are shown. In position 1 the UMD is in a radial position. In position 2, the UMD is nearly in an axial position. In general, as the UMD's position approaches an axial position, $\|\overline{\mathbf{f}}\|$ approaches 0 for all rotation speeds. In any position, $\|\overline{\mathbf{f}}\|$ asymptotically approaches 0 as $\left\|\boldsymbol{\omega}_{\mathrm{h}}\right\|$ increases. Detailed discussion on the influence of the UMD's position on the magnetic force can be found in [24].

\section{EXPERIMENTAL RESULTS AND DISCUSSION}

In our experiments, the actuator magnet is positioned with a Yaskawa Motoman MH5 6-DOF robotic manipulator [see Fig. 4(a)], and consists of a cylindrical $25.4 \mathrm{~mm}$ diameter, $25.4 \mathrm{~mm}$ long, Grade-N42, diametrically magnetized (i.e., along the diameter) $\mathrm{NdFeB}$ permanent magnet [see Fig. 4(b)] 
driven by a Maxon $24 \mathrm{~V}$ A-Max DC motor with an Advanced Motion Controls servo control drive and amplifier. We demonstrate propulsion of rotating UMDs by actuating a rolling spherical device [see Fig. 4(c)], and a threaded capsule-shaped device [see Fig. 4(d)] similar to the design in [13], that produces translational motion along its principal axis from rotation around the same axis. Both UMDs contain a $6.35-\mathrm{mm}$ cubic Grade-N52 NdFeB magnet positioned at the device's center of gravity, with the dipole moment oriented perpendicular to the device's principal axis in the case of the threaded capsule.

The UMDs were constrained during experimentation in a clear PVC lumen with $22.2 \mathrm{~mm}$ inner diameter, lightly lubricated with personal lubricant jelly. Although the PVC lumen is not representative of the human GI system, it is sufficient for our proof-of-concept experiments. (Note that there is experimental evidence to suggest that capsule-shaped UMDs, similar to Fig. 4(d), can be successfully actuated in the human GI system [13], [25].) The UMD position was obtained using a stereo vision system [see Fig. 4(a)] consisting of two Basler A602FC cameras each fitted with a 4.5- mm fixed-focal-length lens producing sub-millimeter tracking accuracy at 30 framesper-second. Although visually tracking a UMD is not clinically relevant, it is sufficient for our proof-of-concept experiments. Existing clinically relevant UMD localization strategies include RF triangulation [26], magnetic methods [27]-[30], and CT scan or X-ray fluoroscopy [31]. Irrespective of the tracking method, the effect of localization uncertainty on the control techniques presented has been studied in [32].

The actuator magnet's dipole moment magnitude $\left\|\mathbf{m}_{\mathbf{a}}\right\|$ was found using a least-squares fit of the measured field to the pointdipole model. The magnetic field was measured with a custommade three-axis sensor shown in the corner of Fig. 5(a) and (b), constructed using three pairs of Allegro A1301 linear Halleffect sensors mounted on the six sides of a 7 - mm cube. Each sensor has a sensitivity of $25 \mathrm{~V} / \mathrm{T}$ and a range of $\pm 0.1 \mathrm{~T}$. The average value of each sensor pair on two opposing faces approximates the component of the field at the cube's center in the direction normal to the faces. One hundred samples of the field were obtained per millimeter between 30 and $140 \mathrm{~mm}$ from the actuator-magnet center in an axial position, resulting in the fit $\left\|\mathbf{m}_{\mathbf{a}}\right\|=12.7 \mathrm{~A} \cdot \mathrm{m}^{2}$ with $R^{2}=0.999$.

The accuracy of the fit and correctness of the theory were verified by measuring the magnetic field while rotating the actuator magnet in two positions located $100 \mathrm{~mm}$ from the actuator magnet's center. The positions $\mathbf{p}_{1}=[0,100,0]^{\top} \mathrm{mm}$ [see Fig. 5(a)] and $\mathbf{p}_{\mathbf{2}}=[81.61,57.79,0]^{\top} \mathrm{mm}$ [see Fig. 5(b)] are described in the coordinate system depicted at the top of Fig. 5. Fig. 5(c) and (d) shows the measured and predicted magnetic fields and axes of rotation at both positions projected onto the $\mathbf{x z}$ and $\mathbf{y z}$ planes. The actuator magnet rotates at $1 \mathrm{rad} / \mathrm{s}$ in both positions. The predicted $\hat{\omega}_{\mathrm{h}}$, obtained by (6), differed from the measured $\hat{\omega}_{\mathrm{h}}$, obtained using principal-component analysis (the direction of least variance), by $2.94^{\circ}$ for $\mathbf{p}_{1}$ and $0.73^{\circ}$ for $\mathbf{p}_{2}$. Fig. 5 clearly shows that the actuator magnet's field and its axis of rotation are closely predicted by the point-dipole model with $\left\|\mathbf{m}_{\mathbf{a}}\right\|=12.7 \mathrm{~A} \cdot \mathrm{m}^{2}$ in the positions indicated, even though the actuator magnet is nonspherical. The actuator magnet's field is
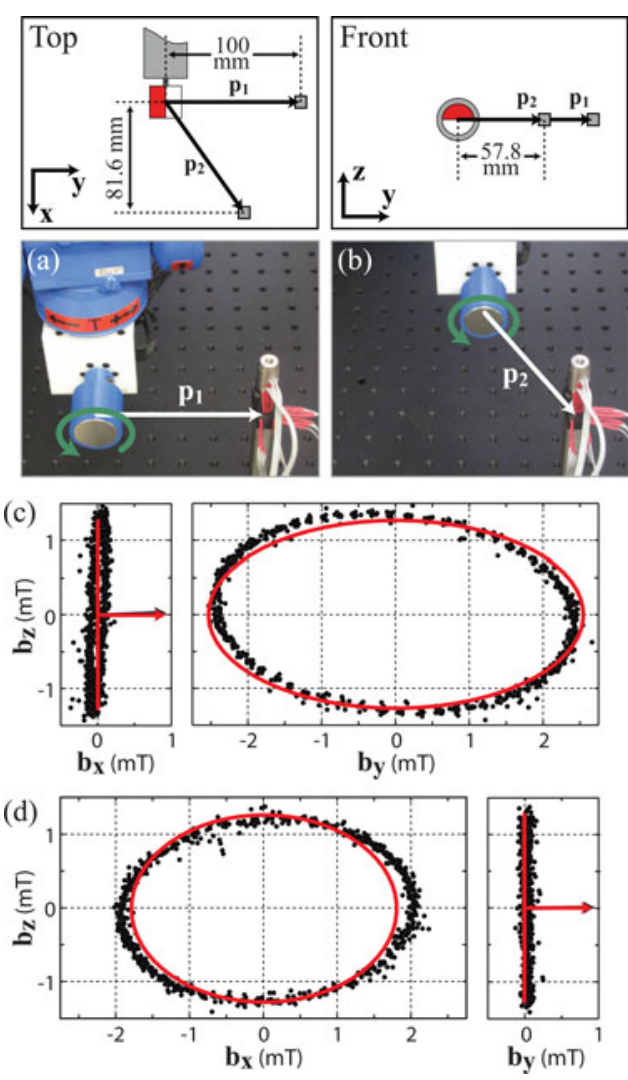

Fig. 5. The components of the magnetic flux density $\mathbf{b}=\mu_{0} \mathbf{h}$ measured and predicted at the positions $\mathbf{p}_{\mathbf{1}}=[0,100,0]^{\top} \mathrm{mm}$ (a) and $\mathbf{p}_{\mathbf{2}}=$ $[81.61,57.79,0]^{\top} \mathrm{mm}(\mathrm{b})$ are shown in (c) and (d), respectively. The predicted $\hat{\omega}_{\mathrm{h}}$ at $\mathbf{p}_{1}$ and $\mathbf{p}_{2}$ differed from the measured $\hat{\omega}_{\mathrm{h}}$ by $2.94^{\circ}$ and $0.73^{\circ}$, respectively. The custom field sensor used to obtain the data appears in the lower right corners of (a) and (b). Note that in the literature, the magnetic flux density is typically denoted as $\mathbf{B}$, however, we denote it in lower-case as $\mathbf{b}$ to maintain the conventions of this paper.

within $3 \%$ of a point-dipole field (3) in every position, provided $\|\mathbf{p}\| \geq 36 \mathrm{~mm}$ [22].

The following experiments were intentionally performed at slow rotation speeds to demonstrate the methods presented herein as purely magnetic effects, with minimal contribution of inertia. Helical UMD propulsion has been previously shown to be robust to misalignment in applied-field rotation axis [32]. The successful actuation of the spherical UMD, which is more sensitive to rotation-axis alignment, will demonstrate that proper control of the rotating magnetic field is achieved.

\section{A. Demonstration Varying $\hat{\boldsymbol{\omega}}_{\mathrm{a}}$, Given $\hat{\boldsymbol{\omega}}_{\mathrm{h}}$ and $\hat{\mathbf{p}}$}

To date, rotating UMDs have been actuated in axial or radial positions where the applied field rotation is easy to visualize and the coupling between the actuator magnet and the UMD is easy to understand. In the radial position, for example, the magnetic field rotates around an axis parallel to that of the actuator magnet, although in the opposite direction. Actuation of the threaded UMD in the radial position is demonstrated in Fig. 6(a). While the UMD travels along the lumen (from right to left), the actuator magnet's position is maintained at $\mathbf{p}=[0,0,-125]^{\top} \mathrm{mm}$ (in the coordinate system depicted in 
(a)
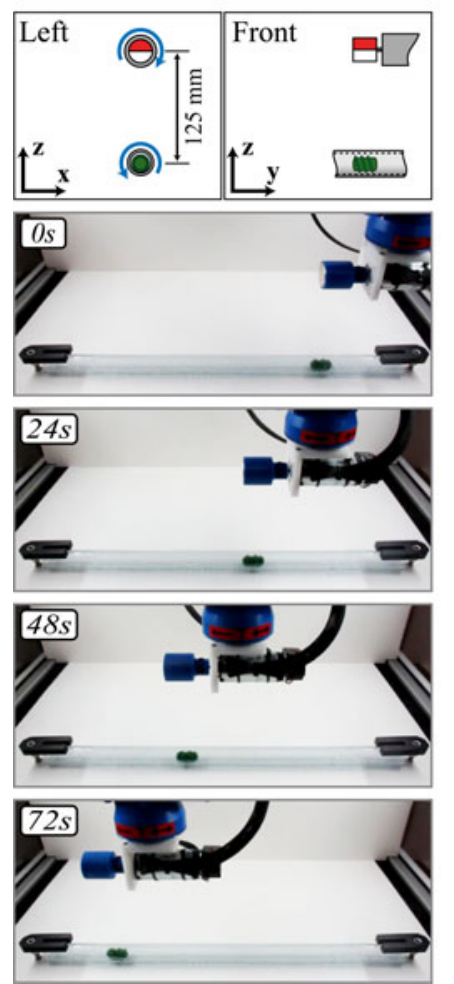

(b)
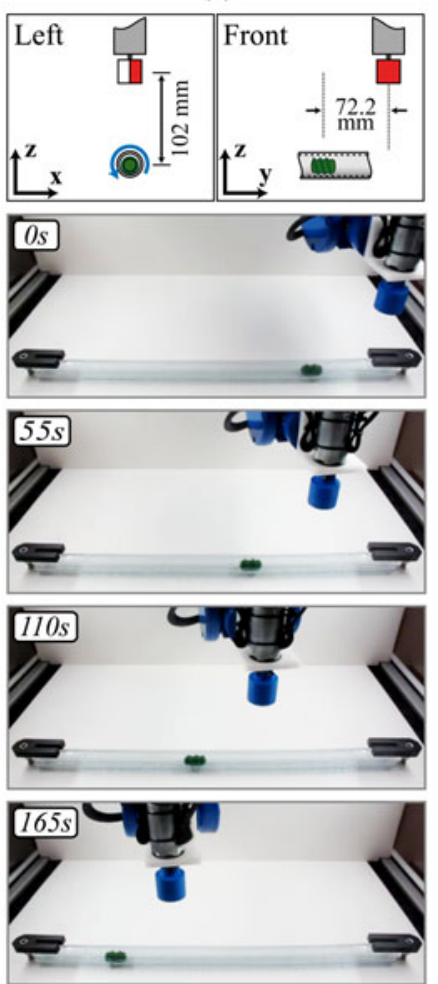

(c)
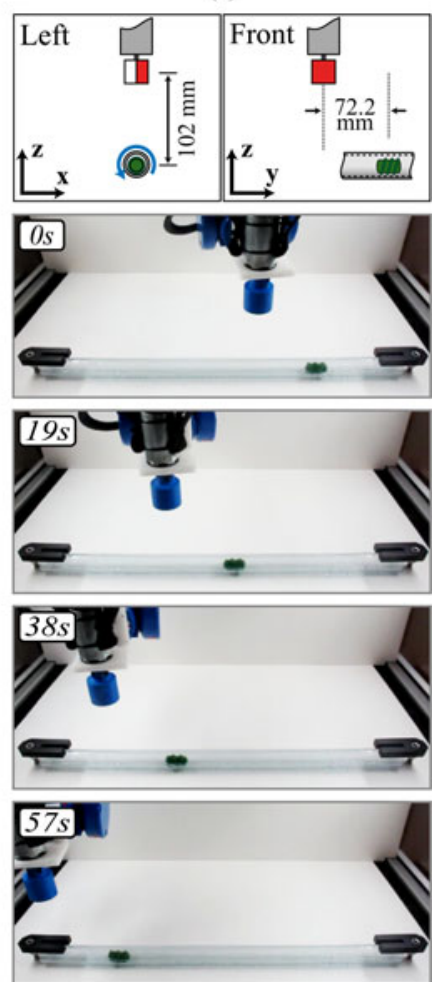

Fig. 6. The threaded capsule UMD is propelled in the (a) radial position with the actuator magnet's position regulated to maintain $\mathbf{p}=[0,0,-125]^{\top}$ mm (in the coordinate system depicted above the captured images) where the necessary actuator-magnet rotation axis is intuitive. Captured images taken during operation with the actuator magnet's position maintained (b) at $\mathbf{p}=[0,-72.2,-102]^{\top} \mathrm{mm}$ and (c) at $\mathbf{p}=[0,72.2,-102]^{\top}$ mm demonstrate the UMD being actuated where the necessary actuator-magnet rotation axis (found by applying (5)) is nonintuitive. In all three examples, $\|\mathbf{p}\|=125 \mathrm{~mm}$ and the actuator magnet was rotated at a constant speed of $\left\|\boldsymbol{\omega}_{\mathrm{a}}\right\|=5 \mathrm{rad} / \mathrm{s}$. The scale drawings above the images depict the scene from direct left and front views. Please see supplementary multimedia.

Fig. 6) using the stereo vision system. Because the external thread converts rotation about the UMD's principal axis to propulsion parallel to its principal axis, $\hat{\omega}_{\mathrm{a}}$ must be parallel to the $y$-axis for propulsion through the lumen. In Fig. 6(a), the UMD travels at an average speed of $3.4 \mathrm{~mm} / \mathrm{s}$. Rotating UMDs actuated in the radial position can be found in [8]-[12].

The theory presented in Section II enables operation of UMDs in nonintuitive ways. Fig. 6(b) shows the threaded UMD actuated with the actuator magnet's position regulated to maintain $\mathbf{p}=[0,-72.2,-102]^{\top} \mathrm{mm}$. After substituting the appropriate $\mathbf{p}$ and $\hat{\omega}_{\mathrm{h}}=[0,-1,0]^{\top}$ into (5), it can be found that the actuator magnet's rotation axis must be $\hat{\omega}_{\mathrm{a}}=[0,0,1]^{\top}$. It is particularly nonintuitive that the necessary actuator-magnet rotation axis is perpendicular to the desired UMD rotation axis in this position. Note that the rotation axis of the field in an equivalent position is measured in Fig. 5(d). The actuator magnet is rotated at $5 \mathrm{rad} / \mathrm{s}$ and causes the UMD to travel from right to left with an average speed of $1.5 \mathrm{~mm} / \mathrm{s}$.

Although the attractive magnetic force must always be managed, it can significantly contribute to propulsion. Fig. 6(c) shows the UMD actuated with $\mathbf{p}=[0,72.2,-102]^{\top} \mathrm{mm}$. In this position, a component of the magnetic force attracts the UMD in the desired direction of motion, producing an average speed of $4.3 \mathrm{~mm} / \mathrm{s}$, which is faster than the average speed in the radial position [see Fig. 6(a)], where the force neither hinders nor helps actuation, and much faster than the position where the actuator magnet trails the UMD [see Fig. 6(b)], where the force hinders actuation. This demonstrates that a rotating UMD can be simultaneously driven and pulled, using the available magnetic torque and force, resulting in faster UMD propulsion. (A threaded UMD in a similar experiment achieved forward velocities of $21.0 \mathrm{~mm} / \mathrm{s}$ in the same configuration [24].)

In Fig. 6, the position of the actuator magnet is regulated to maintain a constant relative position with respect to the UMD. Due to the presence of obstacles in a clinical setting that an actuator magnet must avoid, including the patient, it is unlikely that a UMD will always be actuated in this manner. The theory presented in Section II enables a UMD to be actuated in any position, which may change as the UMD or the actuator magnet move in space, provided that the actuator magnet's rotation axis $\hat{\boldsymbol{\omega}}_{\mathrm{a}}$ is adjusted according to (5). This is demonstrated in Fig. 7 , which shows two examples of the spherical UMD being propelled while the actuator magnet follows a trajectory independent of the UMD. In both examples, the actuator magnet's instantaneous rotation axis $\hat{\omega}_{\mathrm{a}}$ is set according to (5) using the known actuator-magnet position, and the instantaneous UMD position obtained from the stereo vision system, to update the instantaneous position vector p. Fig. 7(a) shows superimposed still images demonstrating the UMD being rolled from left to right while the actuator magnet's position remains stationary. 

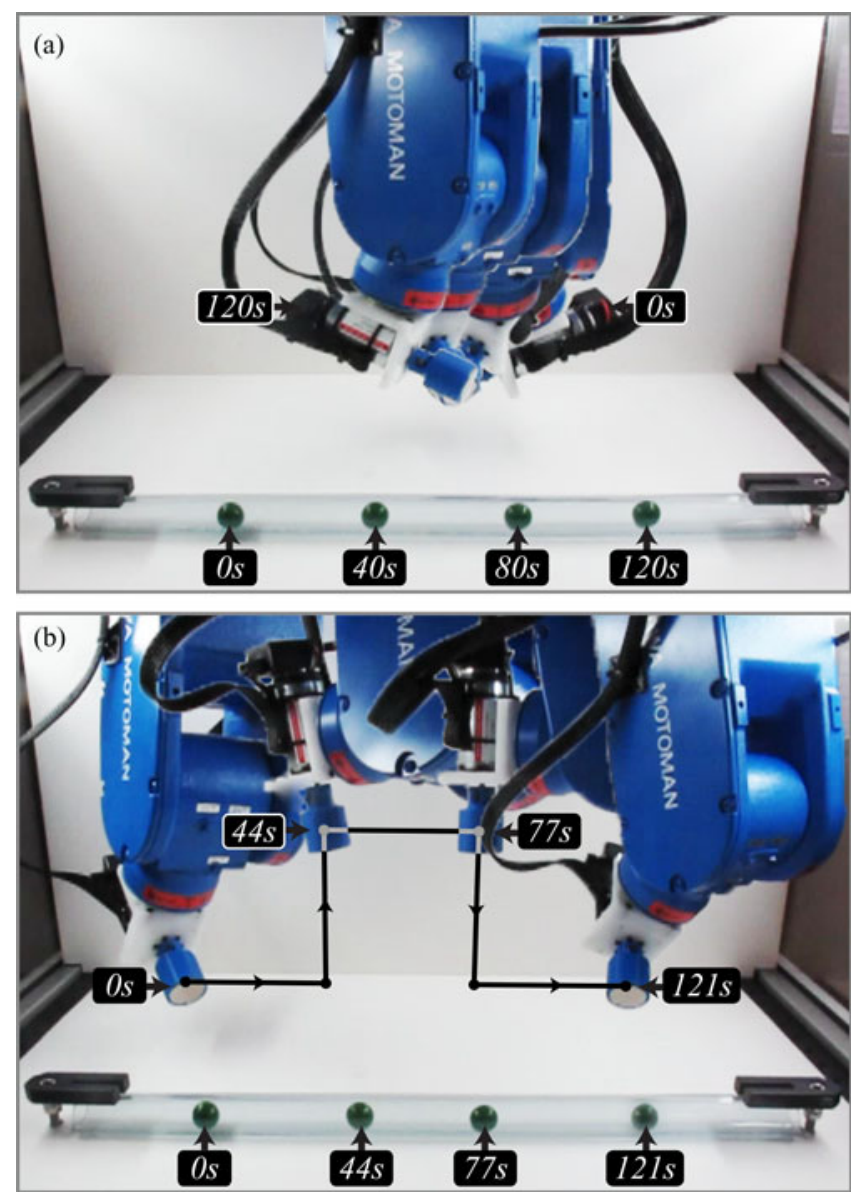

Fig. 7. The spherical UMD [see Fig. 4(c)] is propelled with the actuator magnet's position remaining stationary (a) and following a rectangular step trajectory (b). In both examples, the actuator magnet's position follows the predefined trajectory in an open-loop fashion, independent of the UMD's position. The direction of the actuator magnet's rotation axis $\hat{\omega}_{\mathrm{a}}$ is constantly updated to maintain $\hat{\boldsymbol{\omega}}_{\mathrm{h}}=[-1,0,0]^{\top}$ (in the same coordinate system as Fig. 6) by applying (5), using the known actuator-magnet position and the measured UMD position to update the relative position $\mathbf{p}$. $\left\|\boldsymbol{\omega}_{\mathrm{a}}\right\|=0.25 \mathrm{rad} / \mathrm{s}$ in both cases. Please see supplementary multimedia.

In the case of Fig. 7(b), the UMD rolls from left to right while the actuator magnet follows a rectangular step trajectory. The actuator magnet initially travels parallel to the lumen until it jogs vertically at $t=33 \mathrm{~s}$ with a rate of $9.47 \mathrm{~mm} / \mathrm{s}$; at $t=44 \mathrm{~s}$, the actuator magnet continues parallel to the lumen at $3.16 \mathrm{~mm} / \mathrm{s}$ until $t=77 \mathrm{~s}$, when the actuator magnet moves back to its original height at $9.47 \mathrm{~mm} / \mathrm{s}$; the actuator magnet then finishes moving parallel to the lumen at $3.16 \mathrm{~mm} / \mathrm{s}$. It is easy to imagine this trajectory being used to avoid an obstacle.

In both examples, $\hat{\omega}_{\mathrm{h}}=[-1,0,0]^{\top}$ (in the same coordinate system as Fig. 6) without entering a radial or axial position, and the actuator magnet is rotated at $\left\|\omega_{\mathrm{a}}\right\|=0.25 \mathrm{rad} / \mathrm{s}$. Although both actuator-magnet trajectories are arbitrary, they demonstrate UMD actuation that was not possible using prior methods that only considered axial or radial positions. In practice, actuatormagnet position trajectories should be planned to prevent loss of control authority, employ available magnetic force, avoid collision with obstacles, avoid manipulator singularities or joint limits, and mitigate the influence of localization uncertainty on UMD actuation [32].

\section{B. Demonstration Varying $\hat{\mathbf{p}}$, Given $\hat{\omega}_{\mathrm{h}}$ and $\hat{\boldsymbol{\omega}}_{\mathrm{a}}$}

If the rotation axis of the actuator magnet is constrained such as by the use of a 3-DOF robotic manipulator, then any desired field rotation axis can still be achieved by appropriately selecting the actuator magnet's position (refer to Section II-A2). Although they were originally obtained by setting the actuator magnet's position and solving for the required actuator-magnet axis of rotation, Fig. 6(b) and (c) can also be used to demonstrate the actuation of the capsule UMD while maintaining a constrained actuator-magnet rotation axis. In this example, the desired field rotation axis at the UMD's position is $\hat{\boldsymbol{\omega}}_{\mathrm{h}}=[0,-1,0]^{\top}$, and the actuator magnet's rotation axis is constrained to lie parallel to the $\mathrm{z}$-axis, with the ability to reverse the actuator magnet's direction of spin. The four position solutions, given by (13), that achieve the desired field rotation axis are shown in Fig. 2(a). Two of the four solutions, $\mathbf{p}_{2}$ and $\mathbf{p}_{3}$, place the actuator magnet below the experimental setup's floor and are not physically achievable. The other two solutions, $\mathbf{p}_{1}$ and $\mathbf{p}_{4}$, correspond to the actuatormagnet rotation axes $\hat{\boldsymbol{\omega}}_{\mathbf{a}}=[0,0,-1]^{\top}$ and $\hat{\boldsymbol{\omega}}_{\mathbf{a}}=[0,0,1]^{\top}$, and are shown in Fig. 6(b) and (c), respectively. When multiple feasible solutions exist, the position can be chosen to maximize the contribution of magnetic force to actuation, improve the robustness of actuation to uncertainty in the UMD's location [32], or maximize the configuration-space distance of the robot manipulator from singularities or joint limits.

In the previous example, the desired field rotation axis $\hat{\omega}_{\mathrm{h}}$ remains constant through time and the four actuator-magnet position solutions never change. Fig. 8 shows superimposed still images demonstrating the spherical UMD traveling from left to right along a curved lumen, where the field rotation axis and the actuator magnet's position solutions change. At every instant in time, the position of the actuator magnet is chosen using (13) to insure that the field rotation axis $\hat{\boldsymbol{\omega}}_{\mathrm{h}}$ is always perpendicular to the curved lumen. The desired field rotation axis $\hat{\boldsymbol{\omega}}_{\mathrm{h}}$ is adjusted manually by the operator as the UMD rolls through the lumen. Fig. 8(a) shows the actuator magnet propelling the spherical UMD, while the actuator magnet's rotation axis is kept fixed at $\hat{\omega}_{\mathrm{a}}=[-0.71,0,0.71]$. The case where the actuator magnet's rotation axis is kept fixed at $\hat{\omega}_{\mathrm{a}}=[0,-0.71,0.71]$ is shown in Fig. 8(b). Allowing the actuator magnet's spin direction to be reversed, there are four possible positions where the actuator magnet can be positioned to achieve the desired $\hat{\omega}_{\mathrm{h}}$. Two of the potential positions obtained from (13) result in the actuator magnet being placed below the floor of the experimental setup and are physically unrealizable. One of the two physically realizable positions caused the actuator magnet and manipulator to occlude the UMD from the view of the vision system. The actuator magnet was placed in the remaining position at each time step.

\section{Effects of Scaling}

The actuator magnet's magnetic field scales homothetically, meaning that the field of an actuator magnet, whose dimensions 
(a)
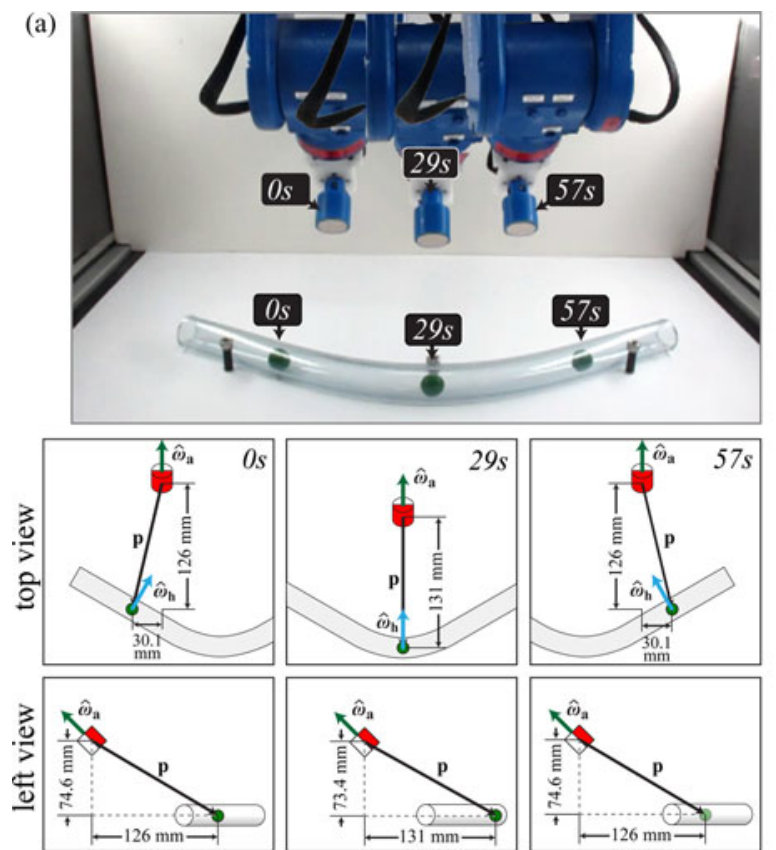
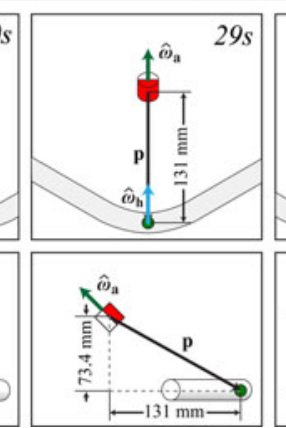
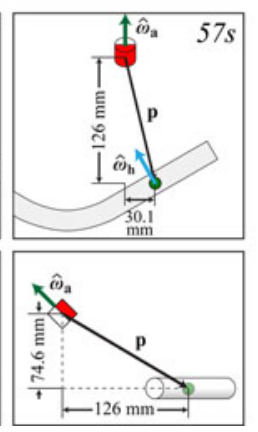

(b)
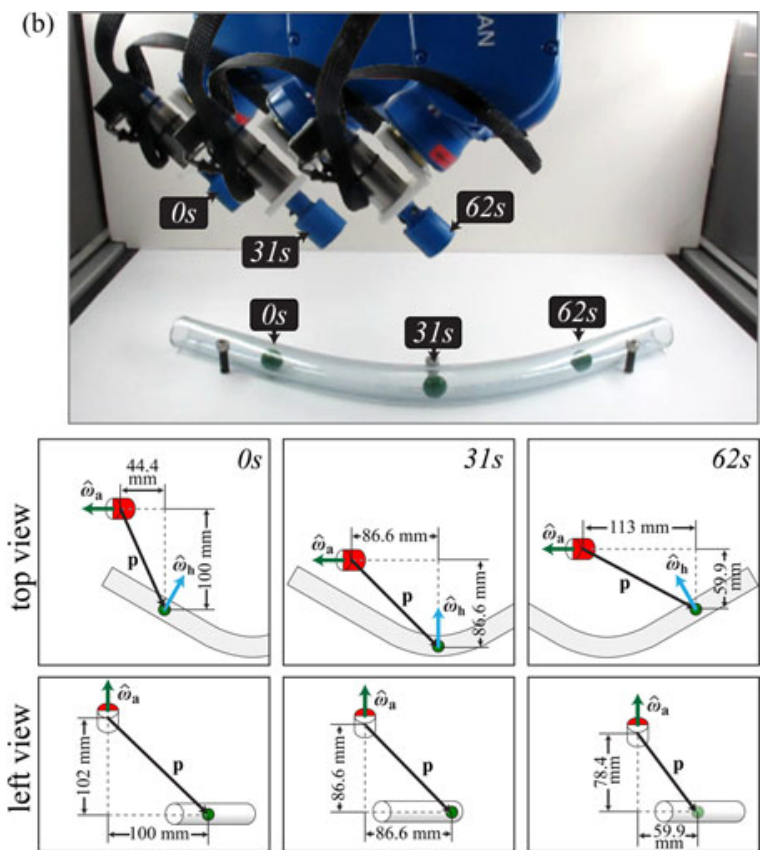

Fig. 8. Spherical UMD [see Fig. 4(c)] is propelled through a curved lumen while maintaining a constant actuator-magnet rotation axis $\hat{\omega}_{\mathrm{a}}$. The magnetic field rotation axis $\hat{\omega}_{\mathrm{h}}$ is kept perpendicular to the curved lumen as the spherical UMD travels. The actuator-magnet poses for three positions of the UMD in the lumen are shown where $\hat{\boldsymbol{\omega}}_{\mathrm{a}}=[-0.71,0,0.71]$ in (a), and where $\hat{\boldsymbol{\omega}}_{\mathrm{a}}=[0,-0.71,0.71]$ in (b). Using the UMD position obtained from the vision system, the actuator magnet's position is adjusted according to (13) to keep $\hat{\omega}_{\mathrm{h}}$ perpendicular to the lumen. Top and side views of each experiment pose are illustrated below (a) and (b). $\|\mathbf{p}\|=150 \mathrm{~mm}$ in all configurations. Please see supplementary multimedia.

have been scaled by a factor $s$, measured at the position $s \mathbf{p}$, is the same as that of the unscaled actuator magnet measured at the position p. This can be verified using (3). Because the dipole magnetization $\left\|\mathbf{m}_{\mathbf{a}}\right\|$ is proportional to the actuator magnet's volume, scaling by $s$ causes the magnetic dipole to become $s^{3}\left\|\mathbf{m}_{\mathbf{a}}\right\|$. Operating the UMD at an equivalently scaled distance away makes $\mathbf{p}$ become $s \mathbf{p}$. After substituting $s^{3}\left\|\mathbf{m}_{\mathbf{a}}\right\|$ and $s \mathbf{p}$ into (3) for $\left\|\mathbf{m}_{\mathbf{a}}\right\|$ and $\mathbf{p}$, respectively, the factor $s^{3}$ appears in both the numerator and the denominator and can be canceled, making the field of the scaled actuator magnet at equivalently scaled distances the same as without scaling. Since the magnetic force is amplified by the scalar $3 \mu_{0}\left\|\mathbf{m}_{\mathbf{d}}\right\|\left\|\mathbf{m}_{\mathbf{a}}\right\| / 4 \pi\|\mathbf{p}\|^{4}$, substituting $s^{3}\left\|\mathbf{m}_{\mathbf{a}}\right\|$ and $s \mathbf{p}$ for $\left\|\mathbf{m}_{\mathbf{a}}\right\|$ and $\mathbf{p}$, respectively, into this amplification factor shows that the magnetic force scales as $s^{-1}$. This implies that when the actuator magnet's dimensions are scaled by $s$ and the UMD is operated at an equivalently scaled distance, the magnetic field (and thus the magnetic torque) remains the same, whereas the magnetic force is diminished. This demonstrates that the magnetic fields generated by the actuator magnet will scale well for clinical use, assuming magnetic torque is the primary means of actuation. However, if the magnetic force is expected to assist actuation [as demonstrated in Fig. 6(c)], then diminishing the force may be undesirable.

\section{CONCLUSION}

To date, rotating UMDs operated using a single rotating permanent magnet have been actuated in positions where the magnetic field behavior is simple and easy to visualize. Constraining the actuator magnet to these positions, however, may preclude the use of rotating-permanent-magnet actuation in clinical set- tings due to the presence of obstacles, including the patient's body. We have demonstrated that a desired rotating magnetic field for UMD propulsion can be generated using a single rotating actuator magnet from any position in space, which enables rotating UMDs to be actuated in any position relative to the actuator magnet, provided the UMD position is known. We have experimentally verified the theory presented in this paper by measuring the magnetic field generated by the rotating actuator magnet, and by propelling a spherical rolling UMD and a threaded capsule UMD in two different scenarios: 1) with the actuator magnet positioned arbitrarily in space and actuator magnet's rotation axis determined in a closed-loop fashion, 2) and with the actuator magnet's rotation axis fixed and the actuator magnet's position determined in a closed-loop fashion. Both scenarios assume the 3-DOF position of the UMD is found with a localization system. Systems that employ our method will not be constrained to actuate in positions where the rotating magnetic field is easy to visualize.

\section{APPENDIX A}

The fact that the rotation axis of the applied magnetic field $\hat{\omega}_{\mathrm{h}}$, the UMD's position $p$, and the rotation axis $\hat{\omega}_{\mathrm{a}}$ of the actuator magnet are coplanar, can be demonstrated by expanding (5):

$$
\hat{\boldsymbol{\omega}}_{\mathrm{h}}=\frac{H^{-1} \hat{\boldsymbol{\omega}}_{\mathrm{a}}}{\left\|H^{-1} \hat{\boldsymbol{\omega}}_{\mathrm{a}}\right\|}=\left(\frac{3\left(\hat{\mathbf{p}}^{\top} \hat{\boldsymbol{\omega}}_{\mathrm{a}}\right)}{2\left\|H^{-1} \hat{\boldsymbol{\omega}}_{\mathrm{a}}\right\|}\right) \hat{\mathbf{p}}-\left(\frac{1}{\left\|H^{-1} \hat{\boldsymbol{\omega}}_{\mathrm{a}}\right\|}\right) \hat{\boldsymbol{\omega}}_{\mathrm{a}}
$$

and thus $\hat{\boldsymbol{\omega}}_{\mathrm{h}}$ is in the span of $\hat{\mathbf{p}}$ and $\hat{\boldsymbol{\omega}}_{\mathrm{a}}$, and it holds that $\hat{\boldsymbol{\omega}}_{\mathrm{h}}, \hat{\mathbf{p}}$, and $\hat{\omega}_{\mathrm{a}}$ are coplanar. It can be likewise shown that the magnetic field $\mathbf{h}$, the UMD's position vector $\mathbf{p}$, and the actuator magnet's dipole moment $\mathbf{m}_{\mathbf{a}}$ are also coplanar. 


\section{APPENDIX B}

An explicit representation of $H^{-1}$ can be found with the identity $H^{2}=H+2 I$, derived using the definition of $H$ and recognizing that $\hat{\mathbf{p}}^{\top} \hat{\mathbf{p}}=1$. Multiplying both sides of $H^{2}$ by $H^{-1}$ produces $H=I+2 H^{-1}$ and subsequently

$$
H^{-1}=\frac{1}{2}(H-I) \text {. }
$$

\section{ACKNOWLEDGMENT}

The authors would like to thank K. Popek for her contribution to the custom 3-DOF magnetic field sensor and D. Cowan for his contributions toward system integration.

\section{REFERENCES}

[1] B. J. Nelson, I. K. Kaliakatsos, and J. J. Abbott, "Microrobots for minimally invasive medicine," Аnпи. Rev. Biomed. Eng., vol. 12, pp. 55-85, 2010.

[2] J. L. Toennies, G. Tortora, M. Simi, P. Valdastri, and R. J. Webster III, "Swallowable medical devices for diagnosis and surgery: The state of the art," J. Mech. Eng. Sci., vol. 224, no. 7, pp. 1397-1414, 2010.

[3] G. Ciuti, P. Valdastri, A. Menciassi, and P. Dario, "Robotic magnetic steering and locomotion of capsule endoscope for diagnostic and surgical endoluminal procedures," Robotica, vol. 28, no. 2, pp. 199-207, 2010.

[4] M. Salerno, R. Rizzo, E. Sinibaldi, and A. Menciassi, "Force calculation for localized magnetic driven capsule endoscopes," in Proc. IEEE Int. Conf. Robot. Autom., 2013, pp. 5334-5339.

[5] M. P. Kummer, J. J. Abbott, B. E. Kratochvil, R. Borer, A. Sengul, and B. J. Nelson, "OctoMag: An electromagnetic system for 5-DOF wireless micromanipulation," IEEE Trans. Robot., vol. 26, no. 6, pp. 1006-1017, Dec. 2010.

[6] G. Lien, C. Liu, J. Jiang, C. Chuang, and M. Teng, "Magnetic control system targeted for capsule endoscopic operations in the stomach: Design, fabrication, and in vitro and ex vivo evaluations," IEEE Trans. Biomed. Eng., vol. 59, no. 7, pp. 2068-2079, Jul. 2012.

[7] G. T. Gillies, R. C. Ritter, W. C. Broaddus, M. S. Grady, M. A. Howard III, and R. G. McNeil, "Magnetic manipulation instrumentation for medical physics research," Rev. Sci. Instrum, vol. 65, no. 3, pp. 533-562, 1994.

[8] M. T. Hou, H.-M. Shen, G.-L. Jiang, C.-N. Lu, I.-J. Hsu, and J. A. Yeh, "A rolling locomotion method for untethered magnetic microrobots," Appl. Phys. Lett., vol. 96, no. 024102, pp. 1-3, 2010.

[9] S. Yim and M. Sitti, "Design and rolling locomotion of a magnetically actuated soft capsule endoscope," IEEE Trans. Robot., vol. 28, no. 1, pp. 183-194, Feb. 2012.

[10] A. W. Mahoney and J. J. Abbott, "Managing magnetic force applied to a magnetic device by a rotating dipole field," Appl. Phys. Lett., vol. 99, no. 134103, pp. 1-3, 2011.

[11] T. W. R. Fountain, P. V. Kailat, and J. J. Abbott, "Wireless control of magnetic helical microrobots using a rotating-permanent-magnet manipulator," in Proc. IEEE Int. Conf. Robot. Autom., 2010, pp. 576-581.

[12] J.-S. Lee, B. Kim, and Y.-S. Hong, "A flexible chain-based screw propeller for capsule endoscopes," Int. J. Prec. Eng. Manuf., vol. 10, no. 4, pp. $27-$ 34, 2009.

[13] A. Chiba, M. Sendoh, K. Ishiyama, K. I. Arai, H. Kawano, A. Uchiyama, and $\mathrm{H}$. Takizawa, "Magnetic actuator for a capsule endoscope navigation system," J. Magnet., vol. 12, no. 2, pp. 89-92, 2007.

[14] A. W. Mahoney, J. C. Sarrazin, E. Bamberg, and J. J. Abbott, "Velocity control with gravity compensation for magnetic helical microswimmers," Adv. Robot., vol. 25, no. 8, pp. 1007-1028, 2011.

[15] L. Zhang, J. J. Abbott, L. X. Dong, B. E. Kratochvil, D. Bell, and B. J. Nelson, "Artificial bacterial flagella: Fabrication and magnetic control," Appl. Phys. Lett., vol. 94, no. 064107, pp. 1-3, 2009.

[16] K. Ishiyama, K. I. Arai, M. Sendoh, and A. Yamazaki, "Spiral-type micromachine for medical applications," J. Micromechatron., vol. 2, no. 1, pp. 77-86, 2003.

[17] A. W. Mahoney, N. D. Nelson, E. M. Parsons, and J. J. Abbott, "Non-ideal behaviors of magnetically driven screws in soft tissue," in Proc. IEEE/RSJ Int. Conf. Intell. Robots Syst., 2012, pp. 3559-3564.

[18] K. Sung Hoon, S, Hashi, and K. Ishiyama, "Methodology of dynamic actuation for flexible magnetic actuator and biomimetic robotics application," IEEE Trans. Magn., vol. 46, no. 6, pp. 1366-1369, Jun. 2010.
[19] E. Paperno, I. Sasada, and E. Leonovich, "A new method for magnetic position and orientation tracking," IEEE Trans. Magn., vol. 37, no. 4, pp. 1938-1940, Jul. 2001.

[20] A. W. Mahoney, D. L. Cowan, K. M. Miller, and J. J. Abbott, "Control of untethered magnetically actuated tools using a rotating permanent magnet in any position," in Proc. IEEE Int. Conf. Robot. Autom., 2012, pp. 33753380.

[21] E. P. Furlani, Permanent Magnet and Electromechanical Devices: Materials, Analysis, and Applications, 1st ed. San Diego, CA, USA: Academic, 2001.

[22] A. J. Petruska and J. J. Abbott, "Optimal permanent-magnet geometries for dipole field approximation," IEEE Trans. Magn., vol. 49, no. 2, pp. 811-819, Feb. 2013.

[23] K. M. Miller, A. W. Mahoney, T. Schmid, and J. J. Abbott, "Proprioceptive magnetic-field sensing for closed-loop control of magnetic capsule endoscopes," in Proc. IEEE/RSJ Int. Conf. Intell. Robots Syst., 2012, pp. 1994-1999.

[24] A. W. Mahoney, S. E. Wright, and J. J. Abbott, "Managing the attractive magnetic force between an untethered magnetically actuated tool and a rotating permanent magnet," in Proc. IEEE Int. Conf. Robot. Autom., 2013, pp. 5346-5351.

[25] H. Zhou, G. Alici, T. D. Than, and W. Li, "Modeling and experimental characterization of propulsion of a spiral-type microrobot for medical use in gastrointestinal tract," IEEE Trans. Biomed. Eng., vol. 60, no. 6, pp. 1751-1759, Jun. 2013.

[26] D. Fischer, R. Schreiber, D. Levi, and R. Eliakim, "Capsule endoscopy: The localization system," Gastrointest. Endoscopy Clin. N. Amer., vol. 14, no. 1, pp. 25-31, 2004

[27] M. Salerno, G. Ciuti, G. Lucarini, R. Rizzo, P. Valdastri, A. Menciassi, A. Landi, and P. Dario, "A discrete-time localization method for capsule endoscopy based on on-board magnetic sensing," Meas. Sci. Technol., vol. 23, no. 1, pp. 1-10, 2012.

[28] M.-G. Kim, Y.-S. Hong, and E.-J. Lim, "Position and orientation detection of capsule endoscopes in spiral motion," Int. J. Prec. Eng. Manuf., vol. 11, no. 1, pp. 31-37, 2010.

[29] C. Di Natali, M. Beccani, and P. Valdastri, "Real-time pose detection for magnetic medical devices," IEEE Trans. Magn., vol. 49, no. 7, pp. 35243527, Jul. 2013.

[30] K. M. Popek, A. W. Mahoney, T. Schmid, and J. J. Abbott, "Localization method for a magnetic capsule endoscope propelled by a rotating magnetic dipole field," in Proc. IEEE Int. Conf. Robot. Autom., 2013, pp. 53285333.

[31] F. Carpi and C. Pappone, "Magnetic maneuvering of endoscopic capsules by means of a robotic navigation system," IEEE Trans. Biomed. Eng., vol. 56, no. 5, pp. 1482-1490, May 2009.

[32] A. W. Mahoney and J. J. Abbott, "Control of untethered magnetically actuated tools with localization uncertainty using a rotating permanent magnet," in Proc. IEEE Int. Conf. Biomed. Robot. Biomechatron., 2012, pp. 1632-1637.

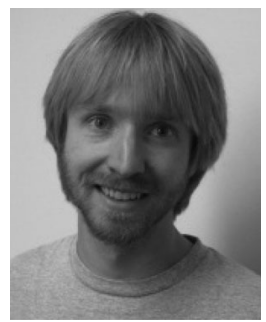

Arthur W. Mahoney (S'10) received the B.S. degrees in computer science and computational mathematics from Utah State University, Logan, UT, USA, in 2009, where he is currently working toward the $\mathrm{Ph} . \mathrm{D}$. degree in computing from the Telerobotics Laboratory.

$\mathrm{He}$ is a National Science Foundation Integrative Graduate Education and Research Traineeship Trainee, a National Science Foundation Graduate Research Fellow, and a Hertz Fellowship finalist. His research interests include the magnetic control of micro- and mesoscale robots.

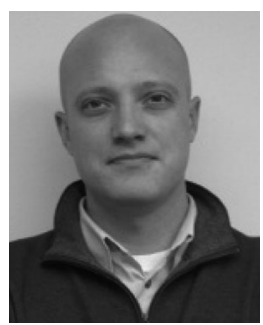

Jake J. Abbott (M'05) received the B.S. degree from Utah State University, Logan, UT, USA, in 1999, the M.S. degree from the University of Utah, Salt Lake City, UT, in 2001, and the Ph.D. degree from Johns Hopkins University, Baltimore, MD, USA, in 2005, all in mechanical engineering.

In 2005, he became a Postdoctoral Research Associate with ETH Zurich, Switzerland. In 2008, he became an Assistant Professor with the Department of Mechanical Engineering, University of Utah, where he is the Head of the Telerobotics Laboratory.

Dr. Abbott received the NSF Faculty Early Career Development Award and the 2010 ICRA Best Manipulation Paper Award. 